\title{
El debido proceso como derecho fundamental en los procesos disciplinarios
} sancionatorios de los estudiantes universitarios

\section{Due process as a fundamental right in disciplinary disciplinary processes of university students}

\author{
Ruth Elizabeth Sánchez-Vintimilla \\ rsanchezv@ucacue.edu.ec \\ Universidad Católica de Cuenca, Cuenca \\ Ecuador \\ https://orcid.org/0000-0001-5232-4099 \\ Cecilia Ivonne Narváez-Zurita \\ inarvaez@ucacue.edu.ec \\ Universidad Católica de Cuenca, Cuenca \\ Ecuador \\ https://orcid.org/0000-0002-7437-9880 \\ Cornelio Agustín Borja-Pozo \\ cborjap@ucacue.edu.ec \\ Universidad Católica de Cuenca, Cuenca \\ Ecuador \\ https://orcid.org/0000-0002-6361-2720 \\ Juan Carlos Erazo-Álvarez \\ jcerazo@ucacue.edu.ec \\ Universidad Católica de Cuenca, Cuenca \\ Ecuador \\ https://orcid.org/0000-0001-6480-2270
}

Recibido: 12 de noviembre de 2019

Aprobado: 17 de diciembre de 2019 


\title{
RESUMEN
}

El debido proceso es un derecho de carácter supra legal, instaurado en la Constitución Política de varios Estados. El derecho a la educación y el principio de autonomía universitaria se complementan entre sí, para inicio a procesos de régimen disciplinario a los estudiantes universitarios cuando el caso amerite, dentro de dichos procesos se encuentran particularidades distintivas por cuanto sus normas no se sitúan en leyes, sino en reglamentos consagrados por las universidades, en el ejercicio del principio de autonomía universitaria, además se encuentra la parte académica de la educación superior y por último la relación estudiante - universidad que se circunscribe al derecho a la educación entendida en su doble dimensión derecho - deber. Este artículo está enfocado a hacer una revisión sobre la cuáles son los elementos que deben contener los procesos sancionatorios emitidos por las Universidades para que garanticen el debido proceso en todas sus etapas.

Descriptores: Derecho fundamental; Debido proceso; Autonomía universitaria; Estudiantes universitarios; Procesos disciplinarios.

\begin{abstract}
Due process is a legal right, established in the Political Constitution of several States. The right to education and the principle of university autonomy complement each other, to start disciplinary procedures to university students when the case warrants, within these processes there are distinctive features because their rules are not placed in laws, but in regulations enshrined by universities, in the exercise of the principle of university autonomy, there is also the academic part of higher education and finally the studentuniversity relationship that is limited to the right to education understood in its double right dimension - must. This article is focused on making a review on what are the elements that must contain the sanctioning processes issued by the Universities to guarantee due process in all its stages.
\end{abstract}

Descriptors: Fundamental right; Due process; University autonomy; University students; Disciplinary processes. 


\section{INTRODUCCIÓN}

El Estado y la sociedad deben garantizar la libertad innata de las personas en cualquier sistema político o económico, manteniendo un debido orden y convivencia social que implica el atribuir varios limites a esta libertad humana evitando el oprimirla, creando leyes que regulen su actuar desde la voluntad popular. El Estado de Derecho y la Constitución son mecanismos creados con el objeto de proteger derechos fundamentales, en este contexto, el Estado debe regular el derecho de su pueblo mas no privarlo del mismo, precautelando el mantener un correcto orden y convivencia social del que se impondrá ciertos límites a la libertad, garantizando la oportunidad de toda persona de acceder de manera imparcial a una justicia social con un trato digno y justo (Duartes, 2018). Cuando una persona comete una infracción o un delito que contraviene las normas jurídicas, es objeto de una sanción o pena, dependiendo del escenario, para llegar a una decisión se debe analizar el caso en concreto y garantizarle a la persona el defenderse de esa acusación.

Para velar que este proceso se lleve a cabo sin vulnerar los derechos de las partes está el principio del debido proceso, puesto que en el trascurso del mismo se puede dar paso a una restricción o privación de un derecho consagrado como fundamental, dicha restricción debe ser legítima, guardando proporcionalidad y razonabilidad con el fin perseguido (lbídem).

En este orden de ideas, la Corte Interamericana de Derechos Humanos (Corte IDH) dentro de su jurisprudencia ha desarrollado al debido proceso como el mecanismo para asegurar progresivamente, el desenlace más ecuánime a una controversia, el mismo que tiene que estar encaminado a para preservar, garantizar 0 afianza el reconocimiento o el goce de un derecho, concluyendo que son parámetros que deben practicarse en razón de una adecuada defensa de las personas cuyos derechos u obligaciones están bajo consideración judicial, debiendo observarse estos requisitos en las instancias procesales (Salmón y Blanco, 2012). 
El debido proceso se caracteriza por ser la realización de una actividad jurisdiccional que se encuentra establecida en la constitución, las leyes y los tratados internacionales suscritos y ratificados por un país, siendo su fin primordial velar por la no violación los derechos de aquella persona que se encuentra encausada en un proceso legal, en el caso de la Constitucion ecuatoriana este derecho se encuentra regulado en el art. 76 (Asamblea Constituyente, 2008).

Partiendo del principio de autonomía universitaria surge el régimen disciplinario que es regulado por las universidades de los países, para ejercer esta potestad se rigen por sus propios estatutos, los mismos que regularan asuntos tanto académicos como disciplinarios, siendo estos de obligatorio cumplimiento para los estudiantes universitarios que han sido matriculados, su desconocimiento o infracción a las mismas implican para el estudiante consecuencias tanto académicas como disciplinarias. Ante este escenario, se debe resaltar que las universidades prestan poca atención a los trámites de los procesos disciplinarios contra estudiantes, lo que ha llevado a la vulneración de derechos fundamentales y a una aplicación inadecuada de un proceso disciplinario sancionatorio (Pabón, Pradilla, y Valencia, 2008).

Sin embargo, en la actualidad se pueden evidenciar que los estudiantes universitarios desconocen cuáles son los elementos que deben tener dichos procesos disciplinarios, si estos están garantizando sus derechos fundamentales plasmados en la constitución, si las universidades están actuando de manera adecuada y eficaz al momento de emitir una sanción, por cuanto las universidades deben ser garantes del cumplimiento ejemplar de la ley, a fin de promover una educación ciudadana integral (Guanipa Ramírez, Albites Sanabria, Aldana Zavala \& Colina Ysea, 2019). Por lo que, el presente artículo tiene por objetivo examinar la línea jurisprudencial sobre el régimen disciplinario sancionatorio en los casos en los cuales se ha pronunciado la Corte Constitucional, a partir de las resoluciones emitidas por las universidades a efectos de garantizar los derechos fundamentales de los estudiantes investigados. 


\section{DESARROLLO}

\section{El derecho a la educación}

La educación es un derecho humano de carácter fundamental, originado con la Declaración Universal de Derechos Humanos en el año 1948, qué, por medio de la Organización de las Naciones Unidas para la Educación, la Ciencia y la Cultura (UNESCO) busca establecer una cultura de paz mediante la cooperación internacional en materia de educación, ciencia y cultura, basado en derechos humanos con el propósito firme de garantizar el goce pleno del derecho a la educación y alcanzar un desarrollo sostenible, procurando igualdad de oportunidades y acceso universal, constituyéndose a lo largo de la vida como un pilar fundamental de los derechos humanos conjuntamente con la democracia, desarrollo local y paz social (García, 2012). Por su parte, la educación superior constituye un instrumento elemental al formar ciudadanos capaces de enfrentar los desafíos del mundo actual promoviendo el desarrollo social mediante la producción de conocimiento, crecimiento económico, fortalecimiento de la identidad cultural nacional, la lucha contra las barreras sociales, contribuyendo a erigir una sociedad más justa, solidaria y promoviendo una cultura de paz con respeto a los derechos humanos (Del Valle, 2019).

Al respecto, la Declaración Universal de los Derechos Humanos en su art. 26 manifiesta que es derecho de toda persona el acceder a la educación de manera gratuita en lo que refiere a la instrucción elemental y fundamental, la misma que será de carácter obligatorio, en cuanto a los estudios superiores puntualiza que el acceso será de manera equivalente para todas las personas en función de los méritos correspondientes (Asamblea General de las Naciones Unidas, 2015).

Además, el Pacto Internacional de Derechos Económicos, Sociales y Culturales en su art. 13 reconoce el derecho de toda persona a acceder a la educación orientada al peno desarrollo de su personalidad y de la dignidad humana, en cuanto a la enseñanza superior señala que debe ser accesible a todos basados en la capacidad de cada uno (Asamblea General de las Naciones Unidas, 1976). Por consiguiente, es deber de los 
Estados el respetar, proteger y promover el acceso efectivo a alcanzar el derecho a la educación como inherente a toda persona humana, la importancia de la educación en si misma radica en la construcción de un pilar fundamental dentro del libre desarrollo de la dignidad y personalidad humana, permitiendo fortalecer el respeto a los derechos humanos y libertades fundamentales (Pérez, 2005).

Dentro de este marco de actuación, la educación debe ser vista mucho más allá de un derecho ya que es un instrumento que permite mejorar la calidad de vida de las personas que acceden a ella. En síntesis, la educación se instituye como una necesidad básica y un derecho humano fundamental que va de la mano con el derecho al desarrollo personal, social y cultural, aportando en gran medida a mejorar la calidad de vida de las personas a través del fortalecimiento de las capacidades y poder de decisión como individuo y miembro de una comunidad, accediendo a una mayor y mejor integración social y cultural que fomenta la participación democrática (García, 2012).

La educación puede ser ofertada por universidades tanto públicas como privadas, las mismas que tienen que garantizar los fines constitucionales válidos; surge entonces el carácter bidimensional de la educación que radica en los privilegios que goza un estudiante en el ejercicio de su derecho constitucional, que a su vez generará en él obligaciones y responsabilidades que cumplir para que el derecho pueda persistir. (Pabón, Pradilla, y Valencia, 2008).

\section{El principio de autonomía universitaria}

Surge como un mecanismo para lograr que las universidades generen una correspondencia entre la libertad académica y su responsabilidad ante la sociedad. En el marco latinoamericano se conoce que en la actualidad diecisiete países de la región la consagran en sus constituciones, entre ellos: Argentina, Bolivia, Brasil, Colombia, Costa Rica, Ecuador, El Salvador, Guatemala, Honduras, México, Nicaragua, Panamá, Paraguay, Perú, República Dominicana, Uruguay y Venezuela. De este modo las instituciones de educación superior se han constituido como un eje clave de aporte al 
desarrollo de países con una visión más crítica, promoviendo un rol sumamente relevante en el crecimiento de las sociedades (Villar, 2014).

Las universidades de la región sobre todo las públicas han sido las principales defensoras del principio de autonomía universitaria, pues comprende: la responsabilidad institucional de organizarse a través de un sistema de cogobierno con la participación activa de los distintos estamentos, la libertad de investigación, la transmisión de conocimientos, la formación con una visión social, la cooperación y apoyo entre las distintas instituciones del sector educativo, y la conservación de vínculos firmes y permanentes con los diversos sectores sociales para transformarse en la conciencia intelectual de la sociedad (Barreto, 2015). La Unión de Universidades de América Latina y el Caribe (UDUAL), en el año 2012 emitió la Declaración de Guadalajara sobre Autonomía Universitaria, en donde se comprometió a fortalecer el Observatorio de Autonomía Universitaria y asumió la tarea de redefinir su concepto para adecuarlo a las circunstancias actuales (Marsiske, 2017).

La autonomía universitaria que ostentan las universidades para emitir sus propios estatutos debe contener también el fundamento legal para llevar a cabo procesos disciplinarios amparados en el principio constitucional del debido proceso. Para ello, es importante que la institución cuente con un reglamento acorde a su realidad educativa, que sea garantista de derechos fundamentales en armonía con la constitución, que en dicho reglamento se especifique la conducta sancionable, que la misma no tenga carácter retroactivo, que el estudiante investigado de manera oportuna conozca las garantías adecuadas para la práctica de su defensa previo a la declaración de una sanción, que la sanción sea acorde y proporcional a la falta cometida. Lo manifestado, permitirá que el procedimiento disciplinario emitido por las universidades en relación a sus estudiantes garantice sus derechos fundamentales amparados en la constitución (Arenas, 2017).

Con fundamento en el principio de autonomía universitaria, se origina el régimen disciplinario que adoptan las universidades con el objeto de tipificar en sus estatutos y 
reglamentos tanto la conducta reprochable como la sanción impuesta, acorde a la garantía del debido proceso. De ello se deriva que, quien goce del derecho a la educación debe actuar acorde a las condiciones previstas para el ejercicio de este derecho, las mismas que abarcan obligaciones de carácter académico, financiero, administrativo y disciplinario, o que producto de su comportamiento infrinja normas internas que regulan relaciones interpersonales atentando a la convivencia institucional, que puede llegar inclusive a imposibilitar el goce de su derecho a la educación (Pabón, Pradilla, y Valencia, 2008).

Es importante recalcar que para un normal desarrollo de la comunidad universitaria es imprescindible contar con reglas de convivencia para llevar a cabo una armonía entre los miembros de la misma, además al ser la educación universitaria considerada como un bien escaso, exige que quienes gocen de este derecho se comprometan al cumplimiento de normas internas de las instituciones a las que pertenecen, estas normas deben estar ajustadas a un correcto funcionamiento de la entidad educativa. Bajo este escenario, los estudiantes pueden utilizar mecanismos adecuados para exigir a las instituciones de Educación Superior la ejecución de sus responsabilidades en la prestación del servicio de la educación, al igual que las universidades pueden solicitar el respeto de las obligaciones a sus estudiantes, con el objetivo de continuar disfrutando de este derecho (Ibídem).

Dentro del marco de la autonomía universitaria surge un elemento de vital importancia al que se conoce como el régimen disciplinario, el mismo que es regulado a través de estatutos, reglamentos y procesos internos. Sin embargo, las universidades no pueden desconocer normas de rango constitucional al momento de establecer sus procedimientos disciplinarios. Es por eso que, el proceso disciplinario se ejercerá por medio de un acto administrativo amparado por el principio del debido proceso (Arenas, 2017). 


\section{La garantía del debido proceso como derecho fundamental}

Con la Declaración Universal de Derechos Humanos se impulsa la evolución del concepto del debido proceso que de forma tradicional ha sido entendido como el procedimiento que en cualquier momento garantiza un trato debido y la protección de derechos civiles y fundamentales de los individuos (Collazos, 2014). La Corte IDH de forma reiterativa ha señalado que el debido proceso surge para proteger, asegurar o hacer valer la titularidad o ejercicio de un derecho (Opinión Consultiva OC-8/87, 1987). Además, el debido proceso es una garantía de los derechos por ende está relacionada con las obligaciones universales que vinculan a los Estados dentro de una sociedad democráticamente constituida.

En Colombia el debido proceso se encuentra plasmado en el art. 29 de la Constitución Política (1991) el mismo que expresa que el principio del debido proceso será aplicable a toda clase de actuaciones judiciales y administrativas; por otra parte, en Ecuador se encuentra estipulado en la Constitución de la República (2008) en el art. 76 que indica que dentro de un proceso en el cual se determinen derechos y obligaciones sea cual fuere el orden, se asegurará el ejercicio del debido proceso con la observación de garantías básicas que se hallan determinadas en siete numerales dentro del mismo artículo; en Perú la observancia del debido proceso se encuentra establecido en el numeral 3 del artículo 139 de su Constitución Política aprobada en el año 1993.

De esta manera, el debido proceso se caracteriza por ser un principio establecido en la Constitución, las leyes y los tratados internacionales suscritos y ratificados por un Estado, cuyo fin primordial es velar por evitar la violación de los derechos de aquella persona que se encuentre encausada en un proceso legal. Razón por la cual, este principio fundamental tiene rango constitucional, y a la vez es un principio general del derecho que puede ser aplicado tanto por el legislador que es el encargado de crear las leyes, como también el juez quien es el que aplica la norma con el objetivo de encontrar la verdad procesal mediante un proceso imparcial (Prieto, 2003). 
El debido proceso es importante dentro de la normativa jurídica y del derecho porque es parte primordial de los derechos humanos, es por esta razón que tiene rango constitucional en las constituciones de distintos países que fundan su organización jurídica y política en un Estado constitucional de derecho. Así mismo, constituye un límite entre la correcta aplicación del derecho y la ilegalidad en el campo de la administración de justicia, lo que trae como consecuencia que los jueces en todas sus actuaciones estén en la obligación de respetar y observar las garantías que lo configuran (Duartes, 2018).

Al respecto, tanto la doctrina como la jurisprudencia se ha pronunciado en razón de que el debido proceso no solo constituye un principio aplicable al ejercicio de la función jurisdiccional sino que además constituye un derecho fundamental, así lo establecen: el artículo 10 de la Declaración Universal de los Derechos Humanos; el artículo XVIII de la Declaración Americana de los Derechos y Deberes del Hombre; el artículo 14 del Pacto Internacional de Derechos Civiles y Políticos; y, el artículo 8 de la Convención Americana sobre Derechos Humanos, en tal contexto, la Corte IDH determina que el debido proceso abarca todas las circunstancias que deben llevarse a cabo con el objetivo de garantizar una apropiada defensa de aquellas personas cuyos derechos $u$ obligaciones se encuentren sujetos a un procedimiento determinado en la ley (Ministerio de Justicia y Derechos Humanos, 2013).

En consecuencia, el debido proceso ha incluido cada vez más importantes garantías y contenidos a la esencia de su definición lo que ha llevado a una innovación dentro de las garantías del debido proceso como lo son el contar con un tribunal imparcial, competente e independiente, el poder ejercer el derecho a la defensa, contar con un plazo razonable, entre otros (Salmón y Blanco, 2012). 


\section{Análisis de la línea jurisprudencial sobre el régimen disciplinario y sancionatorio en las universidades}

En base al principio de autonomía universitaria y debido proceso, surge la potestad sancionadora que pueden ejercer las universidades tanto públicas como privadas, las mismas que por ley se encuentran facultadas a establecer sanciones que garanticen el ejercicio del debido proceso a quien haya cometido una falta u omisión que se encuentre previamente determinada en el cuerpo normativo que la rige (Suárez, Mejía, y Restrepo, 2014). En este marco de referencia, la educación deber ser entendida en su doble dimensión como un derecho - deber del estudiante que es el titular del mismo, concibiendo un modelo educativo de enseñanza aprendizaje que se suma a la obligación académico disciplinaria de reconocer las requerimientos establecidos en cada institución de educación superior, procurando que el actuar del estudiante sea consecuente con los valores y principios institucionales sin llegar a afectar su derecho del libre desarrollo de la personalidad (Amaya, Gómez, y Otero, 2007).

El carácter vinculante del ejercicio de la autonomía universitaria, comprende ciertos límites entre el interés general, orden público y el bien común, es decir, que en las universidades esta facultad no es absoluta puesto que debe adecuarse a los derechos y principios constitucionales, con el objeto de manejar un apropiado control frente a la posibilidad de evitar abusos que en razón del ejercicio de la autonomía universitaria puedan generarse, estas actuaciones pueden ser sujetas a control por parte de la jurisdicción constitucional, cuando su actuar exceda los límites contenidos en la constitución y la ley. En concreto el presente artículo busca revisar los pronunciamientos que forman una línea jurisprudencial, en razón de los procesos disciplinarios desarrollados por las instituciones de Educación Superior, indicando los elementos que deberían contener para garantizar el respeto a los derechos fundamentales de los estudiantes sujetos a un proceso disciplinario (Pabón, Pradilla, y Valencia, 2008). Para este fin se analizarán sentencias emitidas por la Corte Constitucional de Colombia, ya que al realizar una revisión exhaustiva de la literatura 
especialidad, no se identificaron pronunciamientos similares en el resto de países de le Comunidad Andina.

Sentencia fundadora: esta sentencia constituye el primer pronunciamiento emitido por la Corte en la búsqueda de resolver el problema jurídico. En este caso se trata de la Sentencia T-492 de 1992, en la que un estudiante de Comunicación Social solicita a la Universidad en donde cursaba sus estudios le otorgue su título profesional y además le indemnice los daños originados por una sanción de suspensión definitiva emitida en su contra, esto en razón de haber alterado un certificado oficial emitido por la facultad de Comunicación Social para beneficio de un subsidio educativo de la entidad en la que labora su padre. El solicitante consideró que el proceso sancionatorio vulneró su derecho a la defensa y el debido proceso por cuanto no pudo presentar sus descargos, ya que la sanción impuesta no se encontraba plasmada en el reglamento institucional (Corte Constitucional de Colombia, 1992).

Al respecto, la Corte en sus consideraciones señala que si bien es cierto el principio de autonomía universitaria permite a las instituciones de Educación Superior emitir reglamentos que contemplen normas, faltas, procedimientos y sanciones, no se admite bajo ningún criterio que estas instituciones inicien procesos por faltas que no figuren en sus reglamentos, en el caso concreto se suscitaron estos hechos ya que los procedimientos realizados no constaban en su reglamento lo que no garantizo su derecho a la defensa y debido proceso. Por el hecho de que el estudiante había sido sancionado varios años atrás la Corte considero que para el caso concreto la tutela era improcedente por cuanto los acontecimientos se encuentran consumados. Por lo manifestado al ser improcedente la acción de tutela, la Corte Constitucional no se ocupó de realizar un análisis del proceso disciplinario ejercido por la Universidad, pues a su criterio ese estudio carecería de objeto. (Ibídem). 
Sentencia hito: en este tipo de sentencias la Corte procura definir una sub-regla constitucional que es la interpretación de la norma constitucional en razón de una situación fáctica que luego deberá aplicarse en casos análogos. En este sentido, la Corte en la Sentencia T-301 de 1996, estudia los fallos de instancia que resolvieron el recurso de tutela presentado por un estudiante de posgrado en contra de una universidad privada, que fue sancionado producto de un proceso disciplinario. El caso se trata de un odontólogo estudiante de posgrado quien en primer lugar fue suspendido por un semestre, ya que atendió en su consultorio privado a una paciente de sus prácticas sin la supervisión de su docente tutor, posteriormente fue sancionado con la exclusión por un período de dos años de la universidad, esta vez por atender a otra paciente de prácticas en su consultorio privado nuevamente sin supervisión del docente tutor. La universidad considero aplicar una sola sanción referente a los primeros hechos (Corte Constitucional de Colombia, 1996).

El estudiante por su parte entablo una acción de tutela ya que consideró que ha sido vulnerado su derecho al debido proceso, ya que la universidad en ningún momento le comunicó formalmente el inicio de un proceso disciplinario en su contra, además las mencionadas faltas alegadas por la universidad no se encontraban reglamentadas, el estudiante sancionado admitió que en el primer caso fue irregular el haber realizado el tratamiento sin la supervisión de su docente, sin embargo, la segunda vez ya contaba con la autorización del docente tutor, además por el tratamiento realizado en su consultorio privado no cobró valor alguno y manifestó que en ningún momento fue informado sobre las consecuencias disciplinarias que podrían originarse de los hechos (lbídem).

A criterio de la Corte, estos hechos debían sustanciarse en el marco de las garantías constitucionales a favor de las instituciones de Educación Superior y los estudiantes, prevaleciendo el respeto a la dignidad humana pues la autonomía que rige a las universidades no puede afectar los derechos de rango fundamental, puesto que toda decisión que resulte vulneradora de derechos de mayor jerarquía implicaría ser 
ilegitima. Ante este presupuesto, se debe realizar una ponderación con el objetivo de no limitar derechos fundamentales del estudiante ni restringir el ejercicio de la autonomía universitaria, al respecto, la Corte manifiesta que todo procedimiento universitario que pretenda la carga de una sanción debe respetar el núcleo esencial del derecho al debido proceso (lbídem).

Dicho proceso universitario debe contener un elemento sustancial que determine las faltas disciplinarias y las sanciones pertinentes, esto se refiere a que deben ser definidas con anterioridad y previamente tipificadas en un reglamento que debe ser conocido de manera oportuna por quienes regula; y, un elemento procedimental que determine la manera de proceder con antelación a una sanción (Ver figura 1), el mismo que debe ser claro e incluir garantías del debido proceso a la persona investigada. En este caso la Corte halló que el estudiante a pesar de aceptar y reconocer los hechos, nunca fue informado sobre las posibles consecuencias del mismo, además, no se le comunicó que se lo acusaba, razón por la cual no pudo ejercer un oportuno derecho a la defensa. La sanción impuesta no constaba en el reglamento universitario y el acto no fue motivado, en base a esto la Corte determinó dejar sin efecto el proceso emitido por la Universidad dando como resultado la emisión de sanciones, bajo estas consideraciones, el amparo constitucional no solventó la responsabilidad del procesado sino la constitucionalidad del proceso al que fue sometido a fin de resolver su responsabilidad. 


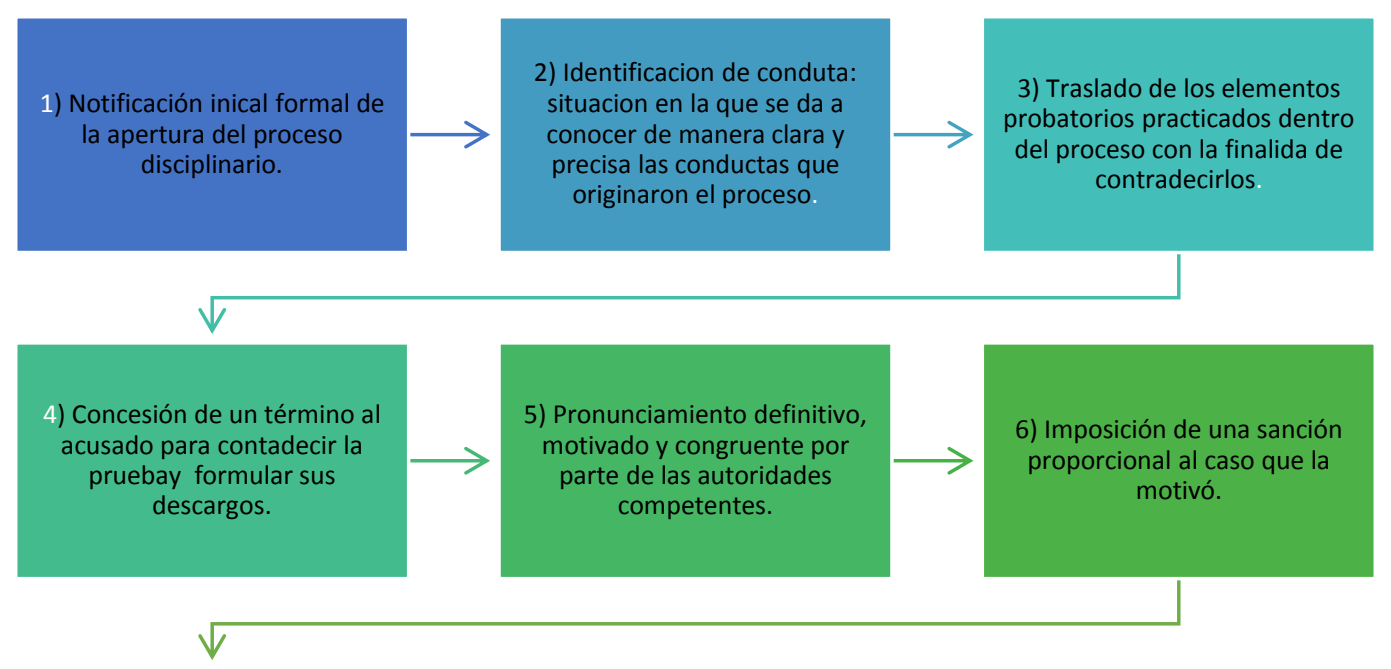

7) Probabilidad de que el imputado alcance a dilucidar mediante recursos adecuados,

las decisiones emitidas por las

autoridades competentes.

Figura 1. Garantías mínimas que deben contener los procedimientos sancionatorios de las universidades

Sentencia arquimédica: esta sentencia es el punto de soporte para la construcción de la línea jurisprudencial y debe ser la más reciente en relación con el tema determinado cuyos hechos están vinculados con el problema jurídico que se quiere resolver. En este caso se analiza la Sentencia T- 720 del año 2012 en la que la accionante es una estudiante sancionada por el Consejo de Enfermería por un caso de plagio, quien determina que la estudiante había incurrido en faltas estipuladas en el reglamento interno dando como resultado su expulsión.

La estudiante sancionada interpuso un recurso de apelación y posteriormente un recurso extraordinario de revocatoria directa presentado ante el Rector de la Universidad, manifestando que se había vulnerado su legítimo derecho al debido proceso, en razón de que los hechos por los cuales se originó el proceso disciplinario, no corresponden a los que imponen la sanción de expulsión. La Corte se pronunció entonces en razón de que el fallo emitido por la Universidad carecía de congruencia y 
motivación ya que no demostró un análisis probatorio del caso, ni se motivó las razones por las cuales el Concejo de Enfermería pretendió imputarle faltas adicionales a la estudiante, concluyendo dejar sin efecto la decisión emitida por la Universidad (Corte Constitucional de Colombia, 2012).

\section{METODOLOGÍA}

La presente investigación se oriento en la revisión de documentos bibliográficos en relación al derecho a la educación, principio de autonomía universitaria, al debido proceso y la línea jurisprudencial sobre el régimen disciplinario sancionatorio en las universidades, a través de un análisis de carácter jurídico con un alcance descriptivo, a partir de la revisión de doctrina, jurisprudencia, libros, revistas y demás artículos científicos.

Presentando un análisis en relación a su origen dentro de la creación de organismos internaciones y la promulgación de tratados universales en materia de derechos humanos, esto en correspondencia a las garantías fundamentales innatas al individuo, en el caso concreto dentro de procesos disciplinarios iniciados por instituciones de Educación Superior, concluyendo que pocos son los pronunciamientos en razón de este tema dentro de los países de la Comunidad Andina, hasta el momento solo la Corte Constitucional de Colombia ha sido quien ha fundado una línea jurisprudencial en relación al régimen disciplinario y sancionatorio, planteando las garantías mínimas que debe contener un proceso disciplinario al interior de las universidades en observación al principio del debido proceso constitucional (Robles, Erazo, Trelles y Narváez, 2020)

\section{APORTES GENERADOS}

La educación es un derecho humano de carácter fundamental, reconocida en el derecho internacional y constitucional, la misma que se encuentra orientada al ejercicio del libre desarrollo de la personalidad. El debido proceso por su parte es una garantía 
fundamental, establecida en la Constitución Política de varios Estados, quienes lo han ratificado por medio de tratados internacionales en materia de derechos fundamentales. Existe una potencial tensión entre el ejercicio de la autonomía universitaria en razón de la garantía del debido proceso, en relación a los procesos disciplinarios de estudiantes que son investigados o sujetos de una sanción disciplinaria por una institución de Educación Superior.

En este sentido la Corte Constitucional de Colombia se ha pronunciado en que la autonomía universitaria no puede servir para la violación del derecho a la educación especialmente en lo relativo al principio del debido proceso. Sin embargo, cuando un estudiante irrumpe las normas emitidas por el estatuto o reglamentos internos de una Universidad, esta tiene la facultad de iniciar procesos disciplinarios que pueden llegar inclusive a una expulsión del estudiante, bajo un proceso previo que garantice su presunción de inocencia, principio de legalidad, y los demás derivados del principio del debido proceso.

Con el fin de evitar que las autoridades incurran en arbitrariedades surge una restricción al ejercicio de la autonomía universitaria con relación al respeto por los derechos fundamentales, pudiendo iniciar acciones tendientes a hacer valer sus derechos en otras instancias procesales.

\section{REFERENCIAS CONSULTADAS}

1. Amaya, R., Gómez, M., \& Otero, A. (2007). Autonomía universitaria y derecho a la educación: alcances y límites en los procesos disciplinarios de las instituciones de educación superior. Revista de Estudios Sociales, 158-165.

2. Arenas, A. (2017). El debido proceso en el procedimiento disciplinario de los estudiantes universitarios. caso universidad de Antioquia. Medellín.

3. Asamblea Constituyente. (2008). Constitución de la República del Ecuador. Montecristi, Ecuador.

4. Asamblea General de las Naciones Unidas. (1976). Pacto Internacional de Derechos Económicos, Sociales y Culturales. 
5. Asamblea General de las Naciones Unidas. (2015). Declaración Universal de los Derechos Humanos. Naciones Unidas.

6. Barreto, D. (2015). La Autonomía Universitaria en el Ecuador. ANALES de la Universidad Central del Ecuador, 1(373), 237-260.

7. Collazos, J. (2014). Derecho a a educación, libre desarrollo de la personalidad y debido proceso. . Colombia.

8. Constitución Política Colombiana. (1991). Constitución Política Colombiana. Bogotá, Colombia.

9. Constitución Política del Perú. (1993). Constitución Política del Perú.

10. Corte Constitucional de Colombia. (1992). Sentencia T- 492 Magistrado Ponente: José Gregorio Hernández Galindo. Bogotá, Colombia.

11. Corte Constitucional de Colombia. (1996). Sentencia T- 301. Magistrado Ponente: Eduardo Cifuentes Muñoz. Bogotá, Colombia.

12. Corte Constitucional de Colombia. (2012). Sentencia T- 720. Magistrado Ponente: Luis Ernesto Vargas Silva. Bogotá, Colombia.

13. Corte Interamericana de Derechos Humanos. (1987). Opinión Consultiva OC8/87. El Habeas Corpus bajo suspensión de Garantías (Arts. 27.2, 25.1 Y 7.6 Convención Americana Sobre Derechos Humanos).

14. Del Valle, D. (2019). Integración y conocimiento. La persistencia de un Derecho: un recorrido por los posicionamientos políticos de y entorno a la Conferencia Regional de Educacion Superior, 2. Nucleo de estudios e investigaciones de Educacion Superior del MERCOSUR.

15. Duartes, E. (2018). El debido proceso como un Derecho Humano. En I. d. (INEJ), El debido proceso como un derecho humano (págs. 7-10). Nicaragua: Hispamer.

16. García, G. (2012). El derecho a la educación, incluida la superior o universitaria, y sus obstáculos, incluido el "Plan Bolonia". Nómadas. Critical Journal of Social and Juridical Sciences, 34(2). Recuperado de https://www.redalyc.org/articulo.oa?id=181/18126057008 
17. Guanipa Ramírez, L., Albites Sanabria, J., Aldana Zavala, J., \& Colina Ysea, F. (2019). Educación para la ciudadanía y la democracia: El equilibrio del poder. IUSTITIA SOCIALIS, 4(6), 71-89. doi:http://dx.doi.org/10.35381/racji.v4i6.290

18. Marsiske, R. (2017). La autonomía universitaria en América Latina a 100 años del movimiento estudiantil de Córdova: una agenda de investigación desde México. Universidades. Recuperado https://www.redalyc.org/articulo.oa?id=373/37352102004

19. Ministerio de Justicia y Derechos Humanos. (2013). Guía sobre la aplicación del Principio - Derecho del Debido Proceso en los procesos administrativos. Lima, Perú: Hecho el Depósito Legal en la Biblioteca del Perú.

20. Organización de Estados Americanos OEA. (2017). OAS. Recuperado de Enero de 2020, de OAS: https://www.oas.org/es/cidh/prensa/comunicados/2017/133.asp

21.Pabón, A., Pradilla, S., \& Valencia, C. (2008). El debido proceso como derecho fundamental de los estudiantes universitarios en los procesos sancionatorios adelantados por las universidades: un análisis a partir de la jurisprudencia de la corte constitucional colombiana. Prolegómenos - Derechos y Valores, XI(21), 109-121.

22. Pérez, L. (2005). La exigibilidad del derecho a la educación a partir del diseño y la ejecución de las políticas públicas educativas. Revista Estudios SocioJurídicos(9).

23. Prieto, M. (2003). El proceso y el debido proceso. En P. U. Javeriana, Vniversitas (p. 811-823). Bogotá.

24. Robles Fernández, P. A., Erazo Álvarez, J. C., Trelles Vicuña, D. F., \& Narváez Zurita, C. I. (2020). Consejo de participación ciudadana y control social transitorio del Ecuador: Destitución corte constitucional. Iustitia Socialis, 25.

25. Salmón, E., \& Blanco, C. (2012). El derecho al debido proceso en la jurisprudencia de la Corte Interamericana de Derechos Humanos. Lima, Perú.

26. Suárez, D., Mejía, P., \& Restrepo, L. (2014). Procedimientos administrativos sancionatorios. Inventario normativo y de las sentencias de la Corte Constitucional de Colombia. Opinión Jurídica. UNIVERSIDAD DE MEDELLÍN, 139-154. 
Iustitia Socialis. Revista Arbitrada de Ciencias Jurídicas.

Año V. Vol. V. ํ⒈ Edición Especial. 2020

Hecho el depósito de Ley: FA2016000064

ISSN: $2542-3371$

FUNDACIÓN KOINONIA (F.K). Santa Ana de Coro, Venezuela

Ruth Elizabeth Sánchez-Vintimilla; Cecilia Ivonne Narváez-Zurita; Cornelio Agustín Borja-Pozo; Juan Carlos Erazo-Álvarez

27. Villar, A. (2014). La autonomía universitaria, una mirada latinoamericana. Ciudad Universitaria, Mexio: UNICAMP.

(C2020 por los autores. Este artículo es de acceso abierto y distribuido según los términos y condiciones de la

licencia Creative Commons Atribución-NoComercial-Compartirlgual 4.0 Internacional (CC BY-NC-SA 4.0)

(https://creativecommons.org/licenses/by-nc-sa/4.0/). 